\title{
Redistribution of soil organic matter by permafrost disturbance in the Canadian High Arctic
}

\author{
David M. Grewer, Melissa J. Lafrenière, \\ Scott F. Lamoureux, Myrna J. Simpson
}

Content Supplementary material

Citation Grewer, D.M., Lafrenière, M.J., Lamoureux, S.F. et al. Biogeochemistry (for article) (2016) 128: 397. https://doi.org/10.1007/s10533-016-0215-7

This is a supplementary material for an article published in

Biogeochemistry. The final authenticated version of the article is available online at: https://doi.org/10.1007/s10533-016-0215-7.

How to cite TSpace items

Always cite the published version, so the author(s) will receive recognition through services that track citation counts, e.g. Scopus. If you need to cite the page number of the author manuscript from TSpace because you cannot access the published version, then cite the TSpace version in addition to the published version using the permanent URI (handle) found on the record page.

This content was made openly accessible by $U$ of $T$ Faculty. Please tell us how this access benefits you. Your story matters. 


\section{$\underline{\text { Supplementary Material }}$}

Redistribution of soil organic matter by permafrost disturbance in the Canadian High Arctic David M. Grewer ${ }^{\mathrm{a}, \mathrm{b}}$, Melissa J. Lafrenière ${ }^{\mathrm{c}}$, Scott F. Lamoureux ${ }^{\mathrm{c}}$, Myrna J. Simpsonª,*

${ }^{a}$ Department of Chemistry, University of Toronto, 80 St George St, Toronto, ON M5S 3H6, Canada

${ }^{\mathrm{b}}$ Department of Physical \& Environmental Sciences, University of Toronto, 1265 Military Trail, Toronto, ON M1C 1A4, Canada

'Department of Geography and Planning, Queen's University, 99 University Ave., Kingston, ON K7L 3N6, Canada

*Corresponding author: Tel.: +1 416287 7234; fax: +1 416287 7279. E-mail address: myrna.simpson@utoronto.ca 


\section{Table S1}

Solvent extractable biomarker concentrations and biomarker proxies from the Upper Goose (UG) soil profile at Cape Bounty Arctic Watershed Observatory.

\begin{tabular}{|c|c|c|c|c|c|c|}
\hline Sample Depth & $\begin{array}{c}0 \mathrm{~cm} \\
(\mathrm{mg} / \mathrm{g} \mathrm{OC})\end{array}$ & $\begin{array}{c}5 \mathrm{~cm} \\
(\mathrm{mg} / \mathrm{g} \mathrm{OC})\end{array}$ & $\begin{array}{c}15 \mathrm{~cm} \\
(\mathrm{mg} / \mathrm{g} \mathrm{OC})\end{array}$ & $\begin{array}{c}30 \mathrm{~cm} \\
(\mathrm{mg} / \mathrm{g} \mathrm{OC})\end{array}$ & $\begin{array}{c}40 \mathrm{~cm} \\
(\mathrm{mg} / \mathrm{g} \mathrm{OC})\end{array}$ & $\begin{array}{c}65 \mathrm{~cm} \\
(\mathrm{mg} / \mathrm{g} \mathrm{OC})\end{array}$ \\
\hline \multicolumn{7}{|l|}{$n$-alkanes } \\
\hline Long-chain Total & $0.289 \pm 0.008$ & $0.430 \pm 0.014$ & $0.439 \pm 0.034$ & $0.310 \pm 0.016$ & $0.283 \pm 0.009$ & $0.062 \pm 0.006$ \\
\hline Short-chain Total & nd & nd & nd & nd & nd & nd \\
\hline $\mathrm{ACL}_{19-33^{\mathrm{a}}}$ & $26.2 \pm 0.1$ & $26.7 \pm 0.1$ & $26.9 \pm 0.1$ & $26.7 \pm 0.1$ & $26.6 \pm 0.1$ & $26.2 \pm 0.6$ \\
\hline $\mathrm{CPI}_{21-33^{\mathrm{b}}}$ & $4.51 \pm 0.31$ & $6.10 \pm 0.48$ & $7.10 \pm 0.75$ & $5.38 \pm 0.48$ & $5.12 \pm 0.38$ & $2.48 \pm 0.44$ \\
\hline \multicolumn{7}{|l|}{$n$-alkanols } \\
\hline Long-chain Total & $1.722 \pm 0.082$ & $1.760 \pm 0.164$ & $2.030 \pm 0.228$ & $1.068 \pm 0.096$ & $0.915 \pm 0.062$ & $0.061 \pm 0.006$ \\
\hline Short-chain Total & $0.008 \pm 0.001$ & $0.010 \pm 0.003$ & $0.017 \pm 0.003$ & $0.011 \pm 0.004$ & $0.011 \pm 0.004$ & $0.0053 \pm 0.0004$ \\
\hline $\mathrm{ACL}_{16-30^{\mathrm{a}}}$ & $25.3 \pm 0.1$ & $25.0 \pm 0.1$ & $24.8 \pm 0.1$ & $24.7 \pm 0.1$ & $24.7 \pm 0.1$ & $23.6 \pm 0.4$ \\
\hline $\mathrm{CPI}_{20-30}{ }^{\mathrm{b}}$ & $14.1 \pm 2.1$ & $8.83 \pm 1.48$ & $11.2 \pm 1.9$ & $11.6 \pm 1.7$ & $11.6 \pm 1.2$ & $4.16 \pm 0.66$ \\
\hline \multicolumn{7}{|l|}{$n$-alkanoic acids } \\
\hline Long-chain Total & $1.387 \pm 0.052$ & $1.554 \pm 0.079$ & $1.828 \pm 0.183$ & $0.443 \pm 0.031$ & $0.330 \pm 0.013$ & $0.042 \pm 0.004$ \\
\hline Short-chain Total & $0.390 \pm 0.050$ & $0.414 \pm 0.070$ & $0.394 \pm 0.045$ & $0.322 \pm 0.074$ & $0.170 \pm 0.032$ & $0.158 \pm 0.018$ \\
\hline $\mathrm{ACL}_{14-28}{ }^{\mathrm{a}}$ & $22.7 \pm 0.4$ & $22.7 \pm 0.4$ & $22.8 \pm 0.5$ & $21.1 \pm 0.8$ & $21.6 \pm 0.3$ & $18.4 \pm 0.1$ \\
\hline $\mathrm{CPI}_{20-28}{ }^{\mathrm{b}}$ & $4.19 \pm 0.22$ & $4.53 \pm 0.39$ & $5.06 \pm 0.87$ & $4.71 \pm 0.55$ & $4.63 \pm 0.35$ & $3.48 \pm 0.58$ \\
\hline \multicolumn{7}{|l|}{ Steroids } \\
\hline Sterols $^{c}$ & $1.357 \pm 0.082$ & $1.522 \pm 0.143$ & $2.187 \pm 0.342$ & $1.617 \pm 0.216$ & $1.414 \pm 0.172$ & $0.038 \pm 0.005$ \\
\hline Sterones $^{\mathrm{d}}$ & $0.318 \pm 0.014$ & $0.534 \pm 0.042$ & $0.568 \pm 0.069$ & $0.294 \pm 0.031$ & $0.264 \pm 0.018$ & nd \\
\hline Cholesterol & $0.067 \pm 0.002$ & $0.069 \pm 0.014$ & $0.061 \pm 0.004$ & $0.054 \pm 0.003$ & $0.044 \pm 0.003$ & nd \\
\hline Ergosterol & $0.063 \pm 0.020$ & $0.063 \pm 0.024$ & $0.053 \pm 0.008$ & $0.038 \pm 0.010$ & $0.039 \pm 0.008$ & nd \\
\hline \multicolumn{7}{|l|}{ Sugars } \\
\hline Glucose & $0.595 \pm 0.058$ & $0.114 \pm 0.026$ & $0.048 \pm 0.007$ & $0.0167 \pm 0.0002$ & $0.011 \pm 0.003$ & $0.004 \pm 0.002$ \\
\hline Mannose & $0.583 \pm 0.093$ & $0.122 \pm 0.028$ & $0.044 \pm 0.005$ & $0.016 \pm 0.001$ & $0.011 \pm 0.002$ & $0.004 \pm 0.001$ \\
\hline Sucrose & $0.791 \pm 0.183$ & $0.557 \pm 0.134$ & $0.060 \pm 0.010$ & $0.050 \pm 0.008$ & $0.008 \pm 0.002$ & $0.005 \pm 0.002$ \\
\hline Trehalose & $1.500 \pm 0.153$ & $2.257 \pm 0.201$ & $1.550 \pm 0.150$ & $0.289 \pm 0.019$ & $0.194 \pm 0.010$ & $0.007 \pm 0.002$ \\
\hline
\end{tabular}

${ }^{\mathrm{a}} \mathrm{ACL}_{\mathrm{i}-\mathrm{n}}$, average chain length, $=\Sigma\left(\mathrm{i} \mathrm{X}_{\mathrm{i}}+\ldots+\mathrm{nX} \mathrm{X}_{\mathrm{n}}\right) / \Sigma\left(\mathrm{X}_{\mathrm{i}}+\ldots+\mathrm{X}_{\mathrm{n}}\right)$, where $\mathrm{X}$ is concentration, $\mathrm{i}$ is the minimum carbon chain length, and $\mathrm{n}$ is the maximum carbon chain length.

${ }^{\mathrm{b}} \mathrm{CPI}_{\mathrm{i}-\mathrm{n}}$, carbon preference index, $=\Sigma\left(\mathrm{X}_{\mathrm{i}}+\mathrm{X}_{\mathrm{i}+2}+\ldots+\mathrm{X}_{\mathrm{n}}\right) / \Sigma\left(\mathrm{X}_{\mathrm{i}-1}+\mathrm{X}_{\mathrm{i}+1}+\ldots+\mathrm{X}_{\mathrm{n}-1}\right)$, where $\mathrm{X}$ is concentration, $\mathrm{i}$ is the minimum long-chain carbon chain length, and $\mathrm{n}$ is the maximum carbon chain length.

${ }^{\mathrm{c}}$ Sterols: stigmasterol, campesterol, $\beta$-sitosterol, and stigmastanol.

${ }^{\mathrm{d}}$ Sterones: stigmasta-3,5-dien-7-one, sitosterone, and stigmastan-3-one.

nd $=$ not detected 


\section{Table S2}

Solvent extractable biomarker concentrations and biomarker proxies from the Upper Ptarmigan (UPt) soil profile at Cape Bounty Arctic Watershed Observatory.

\begin{tabular}{|c|c|c|c|c|c|c|}
\hline Sample Depth & $\begin{array}{c}0 \mathrm{~cm} \\
(\mathrm{mg} / \mathrm{g} \mathrm{OC})\end{array}$ & $\begin{array}{c}5 \mathrm{~cm} \\
(\mathrm{mg} / \mathrm{g} \mathrm{OC})\end{array}$ & $\begin{array}{c}15 \mathrm{~cm} \\
(\mathrm{mg} / \mathrm{g} \mathrm{OC})\end{array}$ & $\begin{array}{c}30 \mathrm{~cm} \\
(\mathrm{mg} / \mathrm{g} \mathrm{OC})\end{array}$ & $\begin{array}{c}40 \mathrm{~cm} \\
(\mathrm{mg} / \mathrm{g} \mathrm{OC})\end{array}$ & $\begin{array}{c}70 \mathrm{~cm} \\
(\mathrm{mg} / \mathrm{g} \mathrm{OC})\end{array}$ \\
\hline \multicolumn{7}{|l|}{$n$-alkanes } \\
\hline Long-chain Total & $0.068 \pm 0.006$ & $0.103 \pm 0.004$ & $0.198 \pm 0.012$ & $0.188 \pm 0.006$ & $0.066 \pm 0.002$ & $0.089 \pm 0.006$ \\
\hline Short-chain Total & nd & nd & $0.0081 \pm 0.0003$ & $0.0056 \pm 0.0002$ & $0.0032 \pm 0.0002$ & $0.0047 \pm 0.0006$ \\
\hline $\mathrm{ACL}_{19-33^{\mathrm{a}}}$ & $26.6 \pm 0.1$ & $26.0 \pm 0.1$ & $25.5 \pm 0.1$ & $25.9 \pm 0.1$ & $25.3 \pm 0.1$ & $25.2 \pm 0.2$ \\
\hline $\mathrm{CPI}_{21-33^{\mathrm{b}}}$ & $1.58 \pm 0.28$ & $1.17 \pm 0.09$ & $1.24 \pm 0.15$ & $1.32 \pm 0.08$ & $1.97 \pm 0.12$ & $1.72 \pm 0.19$ \\
\hline \multicolumn{7}{|l|}{$n$-alkanols } \\
\hline Long-chain Total & $0.078 \pm 0.007$ & $0.051 \pm 0.003$ & $0.096 \pm 0.007$ & $0.128 \pm 0.008$ & $0.033 \pm 0.002$ & $0.054 \pm 0.006$ \\
\hline Short-chain Total & $0.0028 \pm 0.0005$ & $0.006 \pm 0.002$ & $0.014 \pm 0.004$ & $0.009 \pm 0.002$ & $0.0072 \pm 0.0005$ & $0.007 \pm 0.002$ \\
\hline $\mathrm{ACL}_{16-30}{ }^{\mathrm{a}}$ & $23.6 \pm 0.1$ & $22.9 \pm 0.2$ & $22.7 \pm 0.3$ & $23.4 \pm 0.3$ & $22.5 \pm 0.1$ & $22.7 \pm 0.2$ \\
\hline $\mathrm{CPI}_{20-30}{ }^{\mathrm{b}}$ & $9.71 \pm 2.06$ & $6.10 \pm 1.07$ & $4.39 \pm 0.86$ & $7.68 \pm 0.67$ & $7.55 \pm 1.45$ & $5.52 \pm 1.26$ \\
\hline \multicolumn{7}{|l|}{$n$-alkanoic acids } \\
\hline Long-chain Total & $0.0019 \pm 0.0003$ & nd & $0.047 \pm 0.004$ & $0.071 \pm 0.006$ & $0.023 \pm 0.002$ & $0.027 \pm 0.002$ \\
\hline Short-chain Total & $0.022 \pm 0.002$ & $0.036 \pm 0.002$ & $0.311 \pm 0.034$ & $0.259 \pm 0.016$ & $0.072 \pm 0.004$ & $0.193 \pm 0.020$ \\
\hline $\mathrm{ACL}_{14-28}{ }^{\mathrm{a}}$ & $17.3 \pm 0.1$ & $16.8 \pm 0.1$ & $17.8 \pm 0.1$ & $18.3 \pm 0.2$ & $18.4 \pm 0.1$ & $17.6 \pm 0.1$ \\
\hline $\mathrm{CPI}_{20-28}{ }^{\mathrm{b}}$ & undefined & undefined & $2.85 \pm 0.68$ & $2.48 \pm 0.48$ & $1.45 \pm 0.32$ & $1.65 \pm 0.31$ \\
\hline \multicolumn{7}{|l|}{ Steroids } \\
\hline Sterols $^{\mathrm{c}}$ & $0.060 \pm 0.007$ & $0.036 \pm 0.006$ & $0.028 \pm 0.004$ & $0.036 \pm 0.002$ & $0.013 \pm 0.001$ & $0.016 \pm 0.004$ \\
\hline Sterones $^{\mathrm{d}}$ & nd & nd & nd & nd & nd & nd \\
\hline Cholesterol & $0.007 \pm 0.001$ & $0.009 \pm 0.001$ & $0.018 \pm 0.003$ & $0.020 \pm 0.002$ & $0.008 \pm 0.001$ & $0.012 \pm 0.002$ \\
\hline Ergosterol & nd & nd & nd & nd & nd & nd \\
\hline \multicolumn{7}{|l|}{ Sugars } \\
\hline Glucose & nd & nd & nd & nd & nd & nd \\
\hline Mannose & nd & nd & nd & nd & nd & nd \\
\hline Sucrose & $0.011 \pm 0.003$ & nd & nd & nd & nd & nd \\
\hline Trehalose & $0.123 \pm 0.035$ & $0.030 \pm 0.004$ & $0.018 \pm 0.004$ & nd & nd & nd \\
\hline
\end{tabular}

${ }^{a} \mathrm{ACL}_{\mathrm{i}-\mathrm{n}}$, average chain length, $=\Sigma\left(\mathrm{iX}_{\mathrm{i}}+\ldots+\mathrm{nX} \mathrm{n}_{\mathrm{n}}\right) / \Sigma\left(\mathrm{X}_{\mathrm{i}}+\ldots+\mathrm{X}_{\mathrm{n}}\right)$, where $\mathrm{X}$ is concentration, $\mathrm{i}$ is the minimum carbon chain length, and $\mathrm{n}$ is the maximum carbon chain length.

${ }^{\mathrm{b}} \mathrm{CPI}_{\mathrm{i}-\mathrm{n}}$, carbon preference index,$=\Sigma\left(\mathrm{X}_{\mathrm{i}}+\mathrm{X}_{\mathrm{i}+2}+\ldots+\mathrm{X}_{\mathrm{n}}\right) / \Sigma\left(\mathrm{X}_{\mathrm{i}-1}+\mathrm{X}_{\mathrm{i}+1}+\ldots+\mathrm{X}_{\mathrm{n}-1}\right)$, where $\mathrm{X}$ is concentration, $\mathrm{i}$ is the minimum long-chain carbon chain length, and $\mathrm{n}$ is the maximum carbon chain length.

${ }^{c}$ Sterols: stigmasterol, campesterol, $\beta$-sitosterol, and stigmastanol.

${ }^{\mathrm{d}}$ Sterones: stigmasta-3,5-dien-7-one, sitosterone, and stigmastan-3-one.

nd $=$ not detected 
Table S3

Solvent extractable biomarker concentrations and biomarker proxies from the Lower Ptarmigan (LPt) soil profile at Cape Bounty Arctic Watershed Observatory.

\begin{tabular}{|c|c|c|c|c|c|c|}
\hline Sample Depth & $\begin{array}{c}0 \mathrm{~cm} \\
(\mathrm{mg} / \mathrm{g} \mathrm{OC})\end{array}$ & $\begin{array}{c}5 \mathrm{~cm} \\
(\mathrm{mg} / \mathrm{g} \mathrm{OC})\end{array}$ & $\begin{array}{c}15 \mathrm{~cm} \\
(\mathrm{mg} / \mathrm{g} \mathrm{OC})\end{array}$ & $\begin{array}{c}30 \mathrm{~cm} \\
(\mathrm{mg} / \mathrm{g} \mathrm{OC})\end{array}$ & $\begin{array}{c}40 \mathrm{~cm} \\
(\mathrm{mg} / \mathrm{g} \mathrm{OC})\end{array}$ & $\begin{array}{c}90 \mathrm{~cm} \\
(\mathrm{mg} / \mathrm{g} \mathrm{OC})\end{array}$ \\
\hline \multicolumn{7}{|l|}{$n$-alkanes } \\
\hline Long-chain Total & $0.488 \pm 0.022$ & $0.256 \pm 0.018$ & $0.212 \pm 0.011$ & $0.216 \pm 0.013$ & $0.249 \pm 0.010$ & $0.416 \pm 0.024$ \\
\hline Short-chain Total & nd & nd & nd & nd & nd & nd \\
\hline $\mathrm{ACL}_{19-33^{\mathrm{a}}}$ & $29.0 \pm 0.1$ & $26.3 \pm 0.1$ & $26.4 \pm 0.1$ & $26.6 \pm 0.1$ & $26.2 \pm 0.2$ & $27.5 \pm 0.1$ \\
\hline $\mathrm{CPI}_{21-33^{\mathrm{b}}}$ & $15.6 \pm 1.4$ & $2.99 \pm 0.52$ & $3.63 \pm 0.50$ & $3.41 \pm 0.48$ & $3.25 \pm 0.23$ & $7.70 \pm 1.01$ \\
\hline \multicolumn{7}{|l|}{$n$-alkanols } \\
\hline Long-chain Total & $0.560 \pm 0.022$ & $0.215 \pm 0.005$ & $0.252 \pm 0.004$ & $0.224 \pm 0.004$ & $0.249 \pm 0.008$ & $0.753 \pm 0.017$ \\
\hline Short-chain Total & $0.004 \pm 0.001$ & $0.017 \pm 0.003$ & $0.014 \pm 0.004$ & $0.013 \pm 0.003$ & $0.021 \pm 0.002$ & $0.016 \pm 0.001$ \\
\hline $\mathrm{ACL}_{16-30}{ }^{\mathrm{a}}$ & $25.2 \pm 0.1$ & $23.8 \pm 0.1$ & $24.2 \pm 0.1$ & $23.9 \pm 0.1$ & $23.8 \pm 0.1$ & $24.6 \pm 0.1$ \\
\hline $\mathrm{CPI}_{20-30}{ }^{\mathrm{b}}$ & $8.00 \pm 0.98$ & $7.18 \pm 0.74$ & $11.4 \pm 1.0$ & $11.74 \pm 1.31$ & $16.6 \pm 2.9$ & $14.0 \pm 1.2$ \\
\hline \multicolumn{7}{|l|}{$n$-alkanoic acids } \\
\hline Long-chain Total & $0.638 \pm 0.034$ & $0.164 \pm 0.011$ & $0.102 \pm 0.006$ & $0.117 \pm 0.009$ & $0.124 \pm 0.011$ & $0.214 \pm 0.015$ \\
\hline Short-chain Total & $0.301 \pm 0.019$ & $0.399 \pm 0.047$ & $0.251 \pm 0.023$ & $0.233 \pm 0.038$ & $0.397 \pm 0.045$ & $0.254 \pm 0.042$ \\
\hline $\mathrm{ACL}_{14-28}{ }^{\mathrm{a}}$ & $21.8 \pm 0.1$ & $18.9 \pm 0.2$ & $18.8 \pm 0.1$ & $19.3 \pm 0.1$ & $18.7 \pm 0.1$ & $20.1 \pm 0.2$ \\
\hline $\mathrm{CPI}_{20-28}{ }^{\mathrm{b}}$ & $5.04 \pm 0.56$ & $3.63 \pm 0.6$ & $3.34 \pm 0.52$ & $3.57 \pm 0.59$ & $3.94 \pm 0.67$ & $4.01 \pm 0.54$ \\
\hline \multicolumn{7}{|l|}{ Steroids } \\
\hline Sterols $^{\mathrm{c}}$ & $1.322 \pm 0.027$ & $0.560 \pm 0.046$ & $0.598 \pm 0.029$ & $0.537 \pm 0.033$ & $0.555 \pm 0.022$ & $1.129 \pm 0.060$ \\
\hline Sterones $^{\mathrm{d}}$ & $0.319 \pm 0.015$ & $0.086 \pm 0.008$ & $0.097 \pm 0.011$ & $0.078 \pm 0.006$ & $0.072 \pm 0.010$ & $0.123 \pm 0.015$ \\
\hline Cholesterol & $0.097 \pm 0.002$ & $0.053 \pm 0.007$ & $0.043 \pm 0.006$ & $0.037 \pm 0.006$ & $0.032 \pm 0.002$ & $0.046 \pm 0.005$ \\
\hline Ergosterol & $0.082 \pm 0.002$ & $0.028 \pm 0.006$ & $0.026 \pm 0.006$ & $0.017 \pm 0.006$ & $0.019 \pm 0.003$ & $0.014 \pm 0.001$ \\
\hline \multicolumn{7}{|l|}{ Sugars } \\
\hline Glucose & $0.178 \pm 0.028$ & $0.019 \pm 0.002$ & $0.015 \pm 0.004$ & nd & $0.009 \pm 0.002$ & $0.0055 \pm 0.0003$ \\
\hline Mannose & $0.194 \pm 0.031$ & $0.020 \pm 0.002$ & $0.009 \pm 0.002$ & nd & $0.007 \pm 0.001$ & $0.004 \pm 0.001$ \\
\hline Sucrose & $1.109 \pm 0.079$ & $0.122 \pm 0.023$ & $0.061 \pm 0.016$ & $0.014 \pm 0.004$ & $0.029 \pm 0.007$ & $0.023 \pm 0.004$ \\
\hline Trehalose & $3.381 \pm 0.196$ & $0.683 \pm 0.096$ & $0.104 \pm 0.010$ & $0.059 \pm 0.019$ & $0.052 \pm 0.009$ & $0.067 \pm 0.010$ \\
\hline
\end{tabular}

${ }^{\mathrm{a}} \mathrm{ACL}_{\mathrm{i}-\mathrm{n}}$, average chain length, $=\Sigma\left(\mathrm{iX}_{\mathrm{i}}+\ldots+\mathrm{nX}\right) / \Sigma\left(\mathrm{X}_{\mathrm{i}}+\ldots+\mathrm{X}_{\mathrm{n}}\right)$, where $\mathrm{X}$ is concentration, $\mathrm{i}$ is the minimum carbon chain length, and $\mathrm{n}$ is the maximum carbon chain length.

${ }^{\mathrm{b}} \mathrm{CPI}_{\mathrm{i}-\mathrm{n}}$, carbon preference index, $=\Sigma\left(\mathrm{X}_{\mathrm{i}}+\mathrm{X}_{\mathrm{i}+2}+\ldots+\mathrm{X}_{\mathrm{n}}\right) / \Sigma\left(\mathrm{X}_{\mathrm{i}-1}+\mathrm{X}_{\mathrm{i}+1}+\ldots+\mathrm{X}_{\mathrm{n}-1}\right)$, where $\mathrm{X}$ is concentration, $\mathrm{i}$ is the minimum long-chain carbon chain length, and $\mathrm{n}$ is the maximum carbon chain length.

${ }^{c}$ Sterols: stigmasterol, campesterol, $\beta$-sitosterol, and stigmastanol.

${ }^{\mathrm{d}}$ Sterones: stigmasta-3,5-dien-7-one, sitosterone, and stigmastan-3-one.

nd $=$ not detected 


\section{Fig. S1}

Photographs from the ALD-impacted area of the Ptarmigan catchment of the Cape Bounty Arctic Watershed Observatory: (A) the exposed Upper Ptarmigan (UPt) scar zone; (B) the Lower Ptarmigan (LPt) sliding zone showing evidence of lateral compression ridge formation. Photographs taken [July 25 2012] (photographer: M.J. Lafrenière)
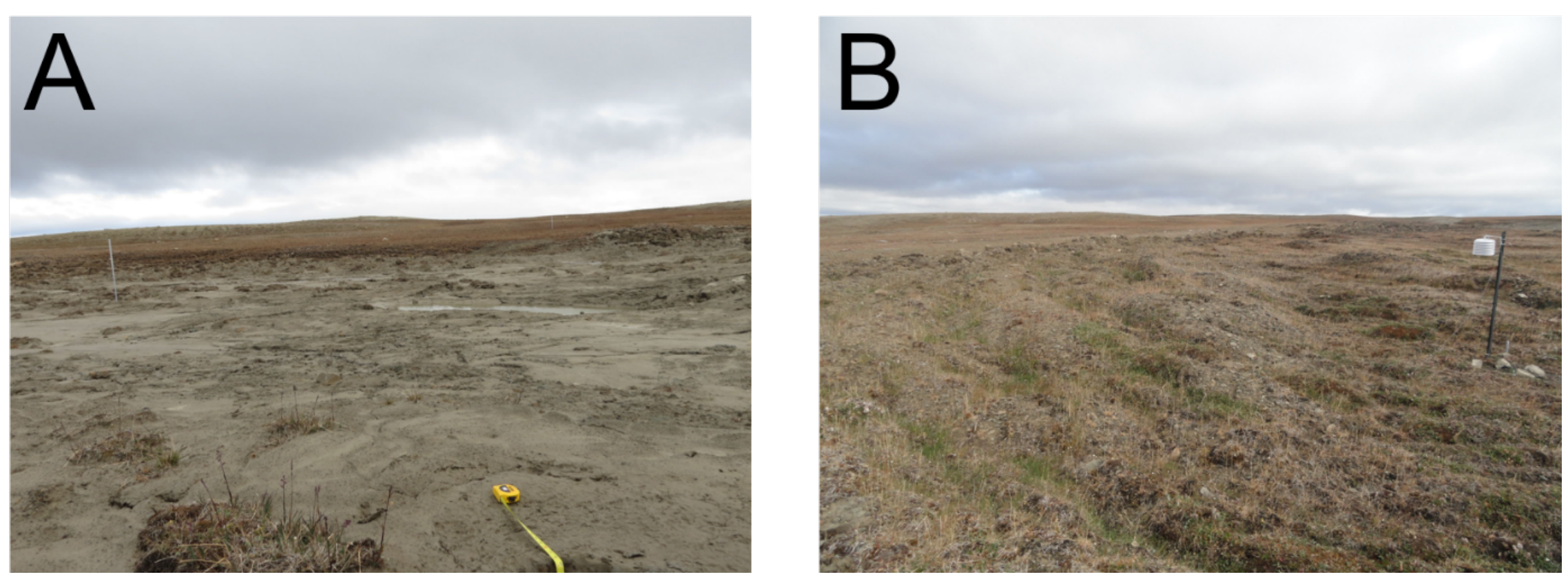
Fig. S2

Distribution of $n$-alkanes extracted from depth profiles of permafrost soil in three study areas of the Cape Bounty Arctic Watershed Observatory.

Upper Goose (UG)
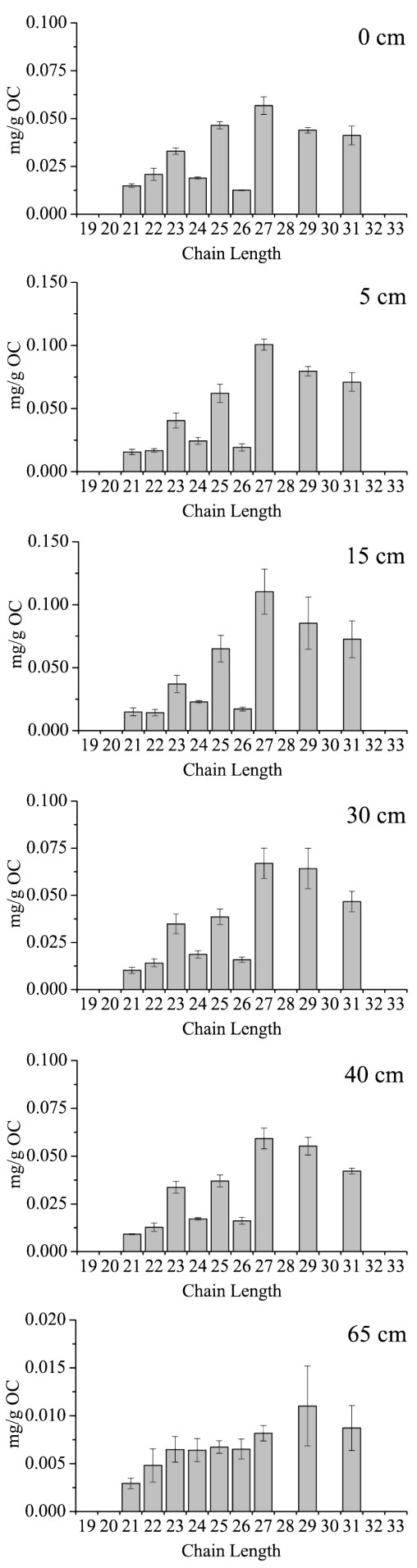

Upper Ptarmigan (UPt)
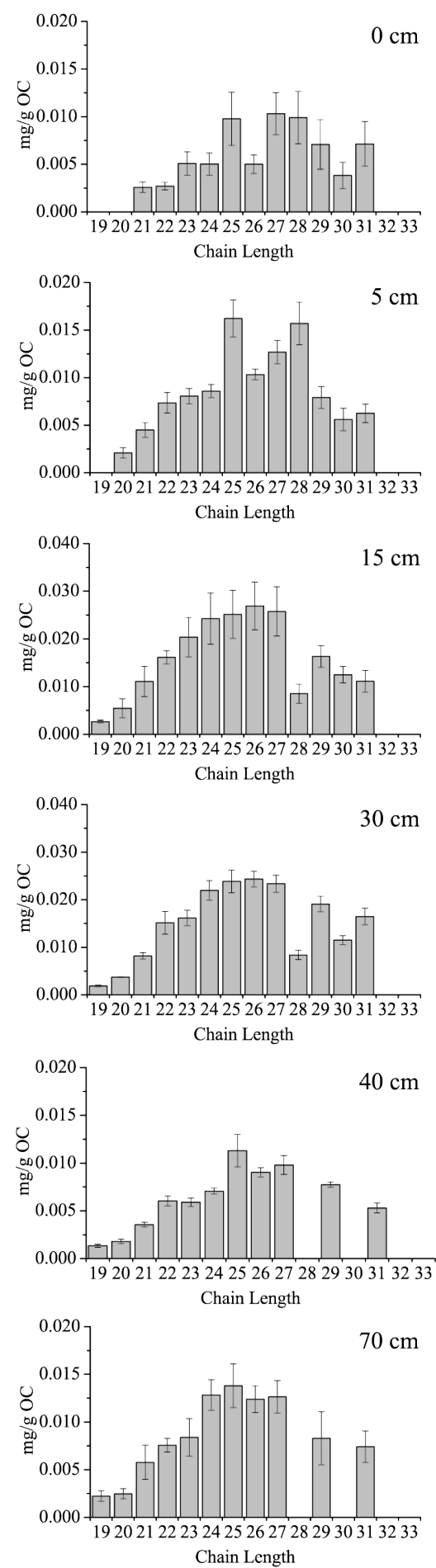

\section{Lower Ptarmigan (LPt)}
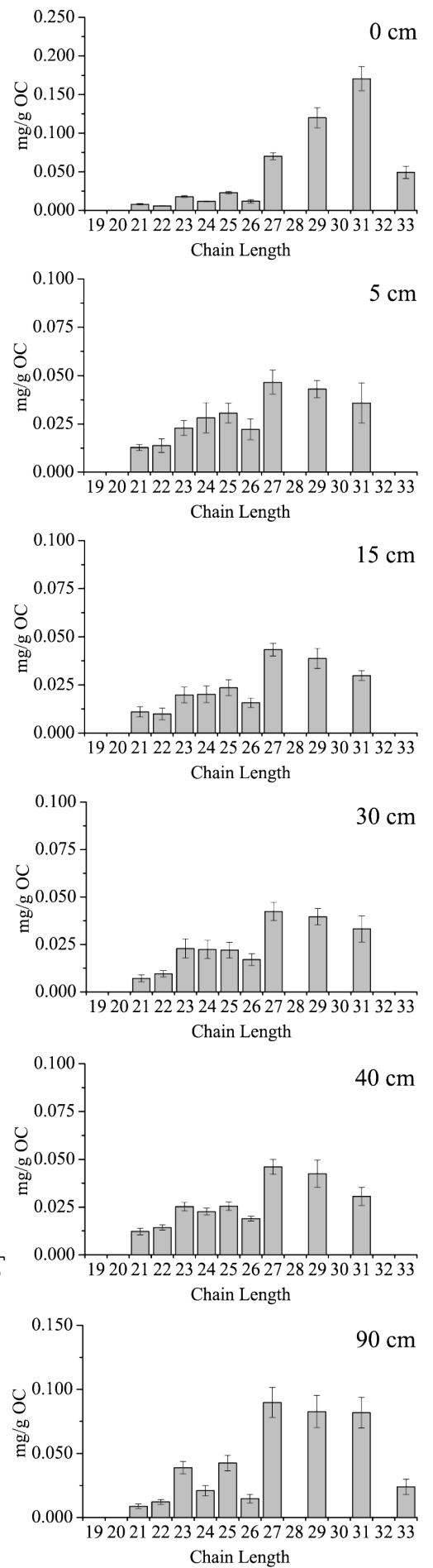
Fig. S3

Distribution of $n$-alkanols extracted from depth profiles of permafrost soil in three study areas of the Cape Bounty Arctic Watershed Observatory.

Upper Goose (UG)
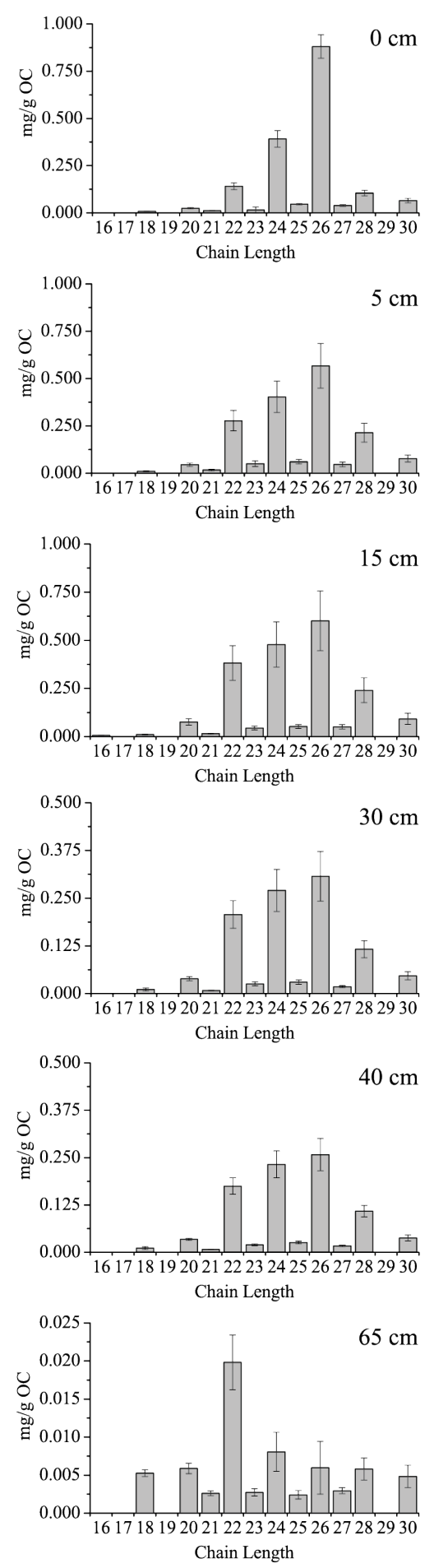

Upper Ptarmigan (UPt)
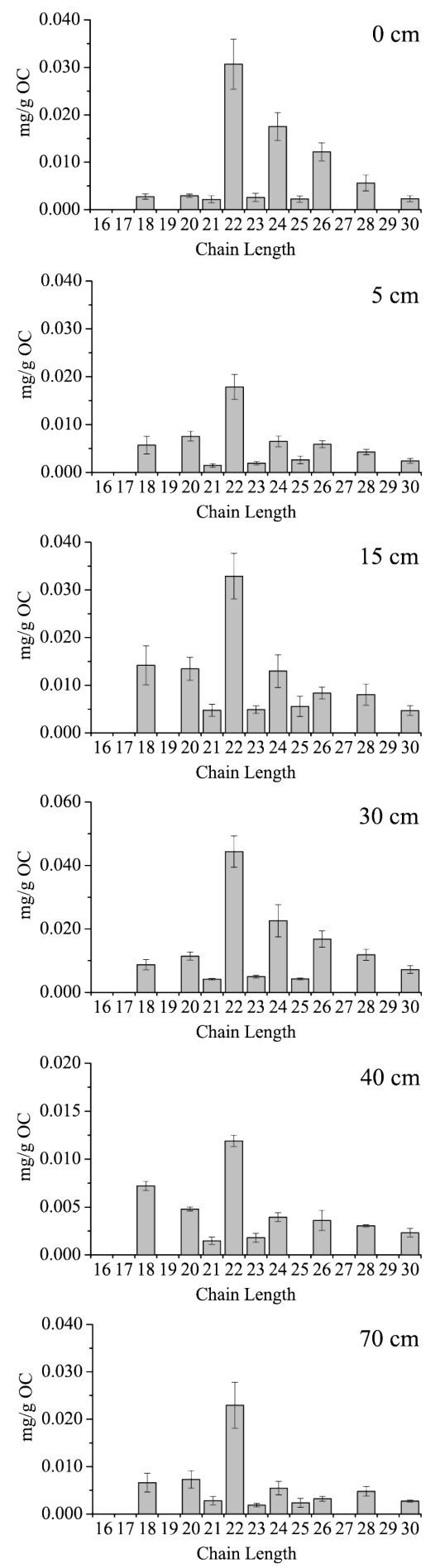

Lower Ptarmigan (LPt)
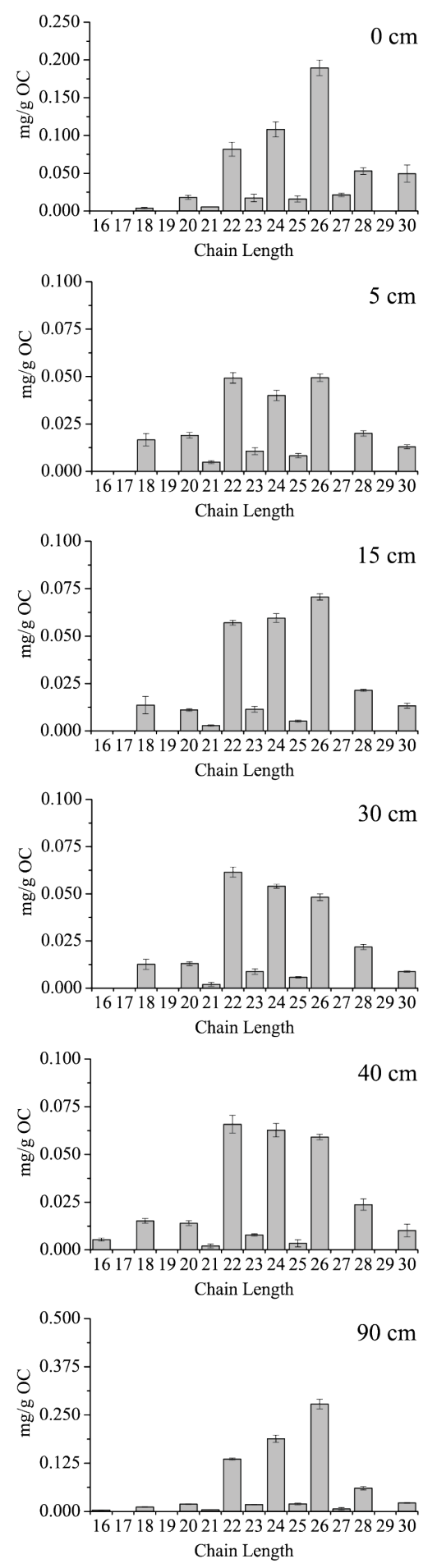
Fig. $\mathbf{S 4}$

Distribution of $n$-alkanoic acids extracted from depth profiles of permafrost soil in three study areas of the Cape Bounty Arctic Watershed Observatory.
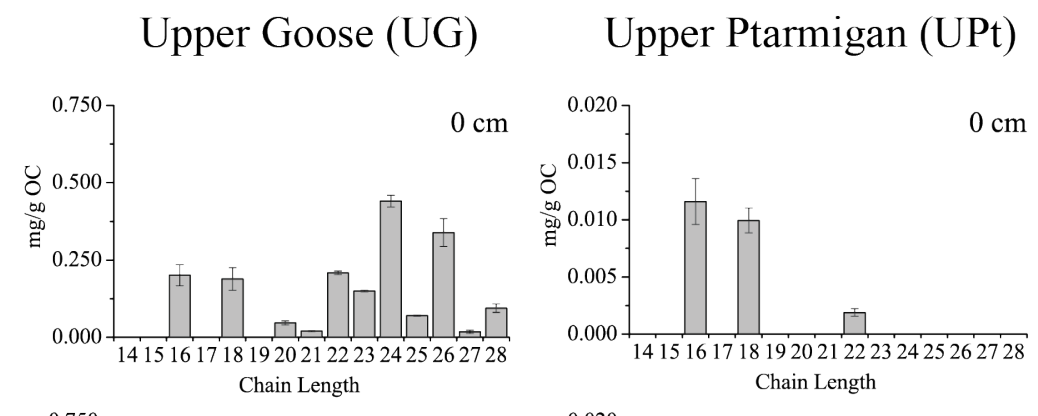

Lower Ptarmigan (LPt)
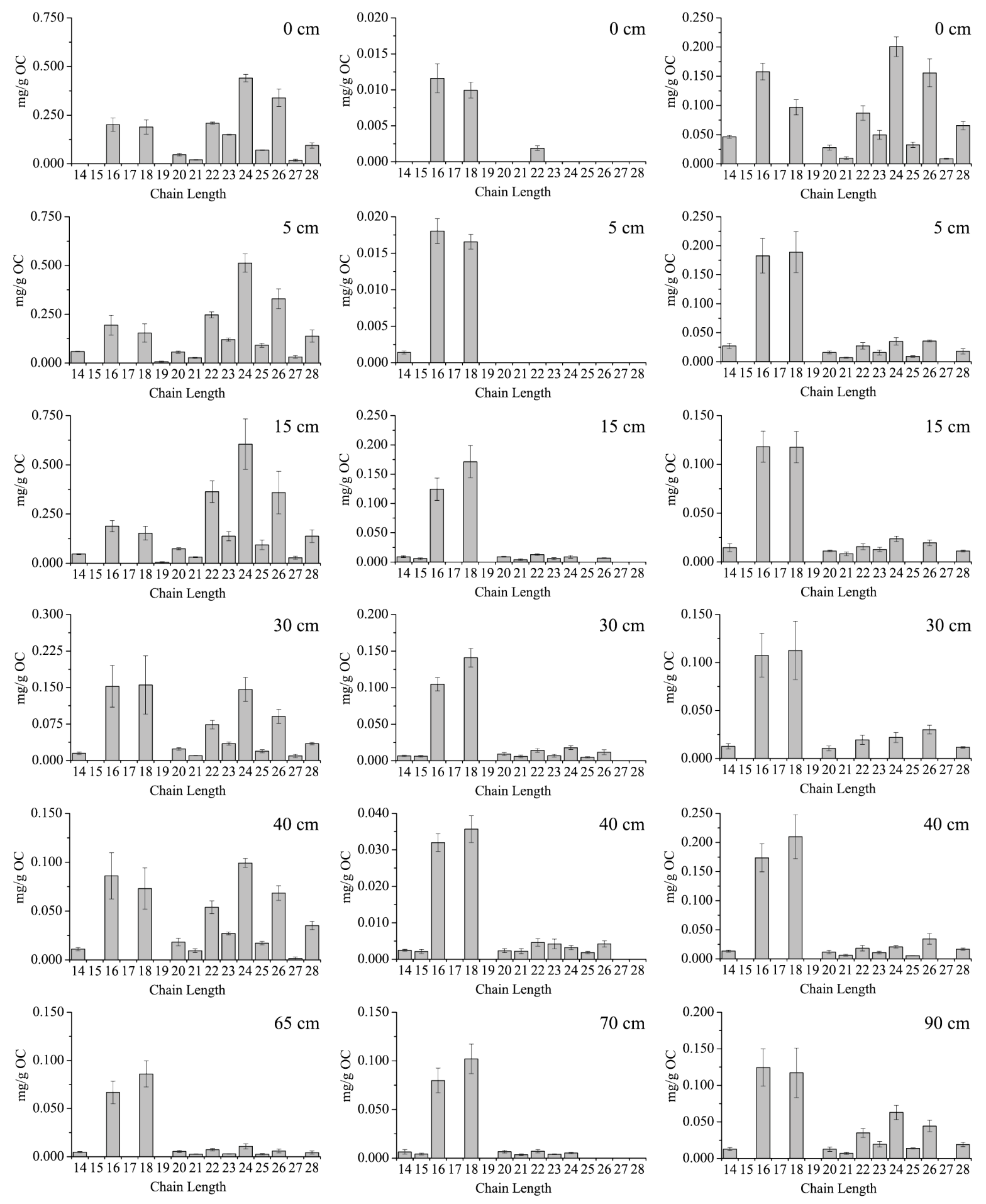